\title{
WAVE DRAG REDUCTION DUE TO A SELF-ALIGNING AERODISK
}

\author{
Ch. Schnepf, O. Wysocki, and E. Schülein \\ German Aerospace Center (DLR) \\ 10 Bunsenstraße, Göttingen 37073, Germany
}

\begin{abstract}
The effect of a self-aligning aerodisk on the wave drag of a blunt slender body in a pitching maneuver has been numerically investigated. The self-alignment was realized by a coupling of the flow solver and a flight mechanics tool. The slender body was pitched with high repetition rate between $\alpha=0^{\circ}$ and $20^{\circ}$ at $\mathrm{M}=1.41$. Even at high $\alpha$, the concept could align the aerodisk to the oncoming flow. In comparison to the reference body without a self-aligning aerodisk, a distinct drag reduction is achieved. A comparison with existing experimental data shows a qualitatively good agreement considering the shock and separation structure and the kinematics of the aerodisk.
\end{abstract}

\section{INTRODUCTION}

One of the most important aerodynamic design goals is the reduction of aerodynamic drag while keeping a high ratio of lift over drag. Considering the Breguet range formula, this leads directly to an increase in range and flight speed. The point of action for the aerodynamic design to reach that goal changes with increasing Mach number due to the different composition of the total drag for different flow regimes. At supersonic and hypersonic speeds, the wave drag dominates. For nonlifting bodies of revolution in these flow regimes, the nose has the largest impact on the drag. The analytical and experimental investigations of [1] showed that for noses with a fixed length, a pointed geometry with a blunt nose tip is most beneficial to minimize the wave drag. The more recent investigation of [2] supports the advantage of the "power law-bodies" in supersonic flight. If aerodynamic heating is considered, then, especially for hypersonic flow, a blunt nose like a semisphere with a large radius $r$ is preferred since the heat flux $\dot{q}$ is reciprocally proportional to the square root of $r$ [3]. For a fixed total body length and diameter, a blunt nose is also beneficial in increasing the space to integrate components like avionic. The total length of the body can be reduced with a hemispherical nose. Unfortunately, a blunt nose in supersonic flight is accompanied by a bow shock wave (Fig. 1a). The total pressure loss over the

This is an Open Access article distributed under the terms of the Creative Commons Attribution License 4.0, which permits unrestricted use, distribution, and reproduction in any medium, provided the original work is properly cited. 


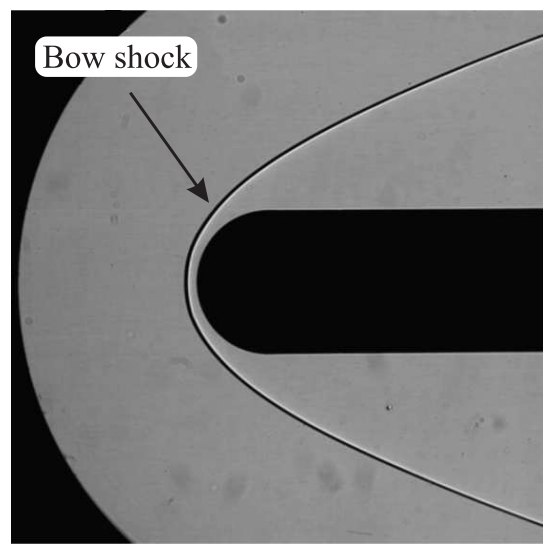

(a)

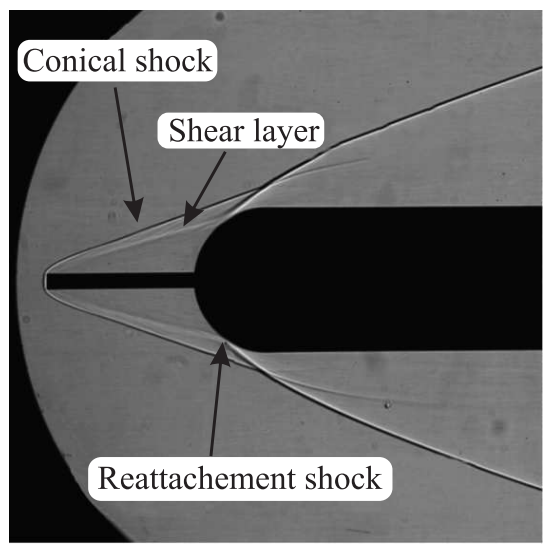

(b)

Figure 1 Shadowgraphs of the bow shock and of the spike-induced separation on a hemispherical model at $\mathrm{M}=5$ and $\alpha=0^{\circ}$ [4]: (a) without spike; and (b) with spike

shock leads to a high wave drag. Here, the loss in total pressure is increased with increasing Mach number.

A well-known concept for reducing the impact of the bow shock on a slender body, while keeping a blunt nose, is the aerospike [5]. The simplest aerospike design is a thin rod mounted on the tip of a blunt body as in Fig. $1 b$.

Slight variations on the initial design include cones, spheres, or disks that are mounted on the tip of the rod. The beneficial effect on the drag has been long known $[6,7]$ and has already been applied in missiles like Trident C-4 und C-5. Keeping the flow between the nose and the spike laminar also results in a significant reduction in heat transfer rate [7].

There are several alternative concepts to the aerospike exist. The concepts range from energizing the air in front of the blunt body due to laser heating (see, e.g., [8-10]), DC-Arc discharge (see, e.g., [11]), or with a wire pointing upstream and heated electrically [12]. Also, the upstream injection of a jet originating from the nose tip showed promising results. Here, jets of air (see, e.g., $[13,14]$ ) or plasma (see, e.g., $[15-17]$ ) were used. The physical principles behind the experiments have been also studied numerically (see, e.g., [18-21]). Most of the concepts induce a similar flow pattern in front of the blunt body to the aerospike. Because of the strong interest in aerospikes and related concepts, several reviews have been published (see, e. g., [5,22]).

For the aerospike in the ideal case, the boundary layer on the rod separates along the whole rod surface due to the pressure rise over the bow shock [23] (see Fig. 1b). The separated boundary layer forms a shear layer that reattaches on the blunt nose. Due to the shear layer, the outer supersonic flow is deflected and 
a weaker conical shock is formed instead of the initial bow shock. A detached bow shock forms just in front of the spike tip. The conical shock unites with the reattachment shock further downstream. A recirculation zone forms inside the shear surface and shows significantly lower pressure levels compared with the blunt body without an aerospike. The investigation of [4] showed that with this simple method, a drag reduction of more than $50 \%$ can be achieved, if the spike and the blunt body show no inclination (angle of attack $\alpha$ ) to the oncoming flow. It has been established that the effectiveness of a spike increases with increasing Mach number. For all Mach numbers, the positive impact of a fixed spike on the wave drag is limited to a range of angle of attack. Even for small $\alpha$, the effectiveness decreases considerably. The beneficial effect completely disappears between $\alpha=15^{\circ}$ and $20^{\circ}$. This is due to the transformation of the favourable symmetric shock system (see Fig. 1b) to an asymmetric shock system like that in Fig. $2 a$.

A strong bow shock reestablishes on windward part of the hemisphere. Additionally, the bow shock interacts with the shock that originates from the spike leading to an undesired Edney type IV [24] shock-shock interaction. This strong dependence of the ef-

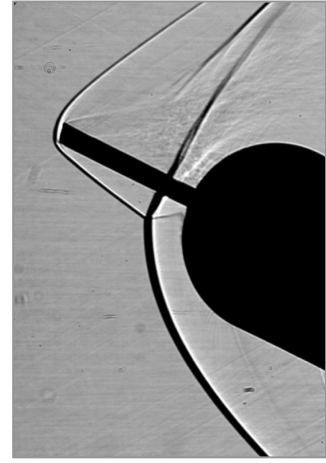

(a)

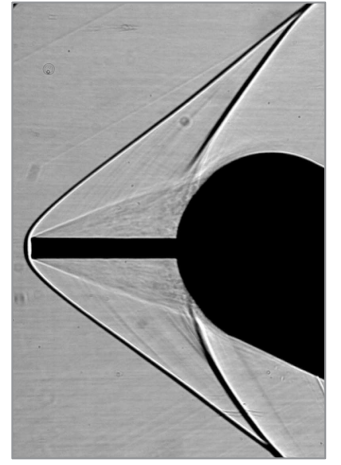

(b)

Figure 2 Shadowgraphies of the flowfield for a fixed $(a)$ and an aligned $(b)$ spikes at $\mathrm{M}=2$ and $\alpha=20^{\circ}[4]$

fectiveness of a spike on $\alpha$ is a huge drawback considering the field of application for some categories of slender bodies. For a modern missile, high maneuverability is a major requirement for some flight missions. Hence, an extension of the range of $\alpha$ where a spike significantly reduces the drag is desirable.

One method is the "self-aligning aerospike" concept [25]. Here, a movable spike points always ideally in the direction of the oncoming flow even though the main body shows an angle of attack. The favorable shock system in front of the nose can be sustained (Fig. $2 b$ ) and the reduction of the pressure drag at the nose is also sustained. The reliability and the effect of a self-aligned aerospike has already been demonstrated in the "proof-of-concept experiment" of [4] for a fixed angle of attack. Due to the alignment of the spike, the decrease of the spike effectiveness is shifted to higher $\alpha$ and the decrease is now moderate. For all tested $\alpha$, the drag could be decreased with the aligned spike. Wysocki et al. [26] adopted the self-aligning spike in their experimental investigation of a generic missile pitching at high frequencies $(0.05-7.5 \mathrm{~Hz})$. In the experiment, the spike 


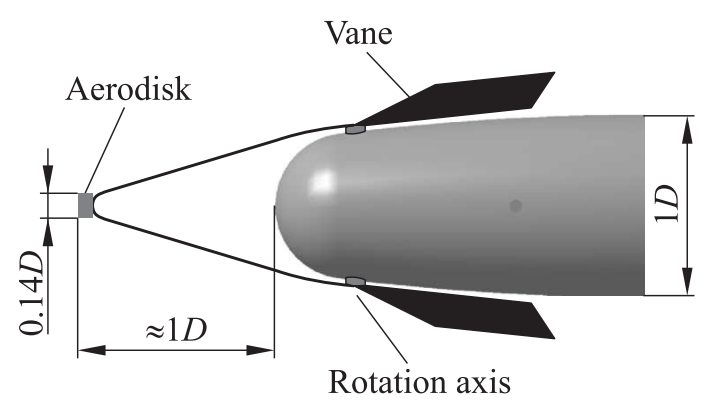

Figure 3 Model nose with self-aligning spike

was replaced with a disk mounted on a frame, rotatable about the nose (Fig. 3). On the other end of the frame, small vanes were attached. In the following sections, this device is abbreviated with "AD." The aerodynamic forces acting on the vanes induced a pitching moment about the hinge and aligned the disk with the oncoming flow. The drag could be reduced successfully for the whole range of angles of attack $\left(0^{\circ}<\alpha<20^{\circ}\right)$ and for all Mach numbers $(1.4<\mathrm{M}<2.2)$ and frequencies.

In agreement with the investigation of [4], the effectiveness increased with increasing Mach number. The present paper is a numerical realization of this version of a self-aligning aerodisk on a pitching slender body. The main motivation of the present investigation is to test the proper functioning of the coupling between the flow solver and the flight mechanic tool for further investigations of a self-aligning aerodisk on a maneuvering missile. Therefore, only a simplified slender body is simulated in comparison to the geometry in [26] to save computational time. The Mach number of the simulation was $M=1.4$. The low supersonic Mach number allowed the physical time steps to be increased. A large reduction of the wave drag at this Mach number cannot be expected considering the investigations of $[4,26]$. Since this paper is dealing with supersonic flow, a qualitative comparison of the flow field at the nose can be made with the corresponding experiment of [26]. In addition to a simulation of the slender body with a self-aligning disk, also, a simulation without a disk has been carried out. The results of both simulations are compared in the present paper.

\section{TEST MODEL GEOMETRY AND COMPUTATIONAL GRID}

The self-aligning disk concept is investigated numerically for the forebody of the generic missile geometry used in the experiments [26]. The computational fluid dynamics (CFD) model geometry consists of a cylinder with a blunt ogive nose (SB), with a total length of $l_{\mathrm{M}}=269 \mathrm{~mm}$ and a diameter of $D=36 \mathrm{~mm}$. The fineness ratio of the nose (hemisphere+ogive) is 1.92. In Fig. 3, the nose with the aerodisk device $(\mathrm{AD})$ is shown. The $\mathrm{AD}$ consists of a disk with a diameter of $d=5 \mathrm{~mm}$. The disk is placed $l=39.3 \mathrm{~mm}$ in front of the blunt nose and is mounted on a frame. Aerodynamic lifting surfaces are mounted on the frame on 


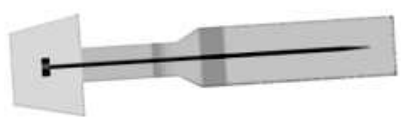

(a)

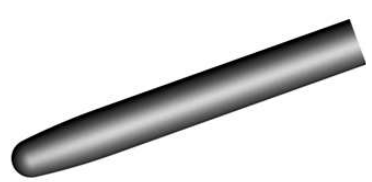

(b)

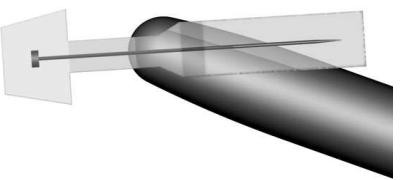

(c)

Figure 4 Chimera grid components: (a) AD geometry with outer grid boundary; (b) SB geometry; and (c) AD and SB superimposed

its right-hand end (see Fig. 3). A joining rod and a hinge for a bearing is not considered in the CFD.

The pitching motion of the $\mathrm{AD}$ about the nose of the $\mathrm{SB}$ is realized with the hole-cutting chimera technique [27]. The chimera technique makes it possible to solve the (unsteady) Reynolds-averaged Navier-Stokes ((U)RANS) equation on overlapping grids. Two grids are required to realize the pitching of the AD about the nose of the SB. One grid contains the geometry of the $\mathrm{AD}$, the other the geometry for the SB (Figs. $4 a$ and $4 b$ ). For the chimera technique, the best quality in interpolation between the grids is achieved if the grids have the same cell size in the overlap area. Therefore, the bottom of the AD grid consists of two layers (Fig. $5 a$ ). The layer closer to the AD geometry shows small cell sizes (L1). This layer is used for the interpolation in the overlap area in the vicinity of the boundary layer of the SB (see Fig. $5 a$ ). Outside this region, L2 is used. To get a proper numerical grid, the grid points of the counterpart grid that are inside the geometry have to be blanked (see Fig. 5). This is achieved with hole definitions. One hole is enveloping the SB geometry and one hole the AD geometry and the

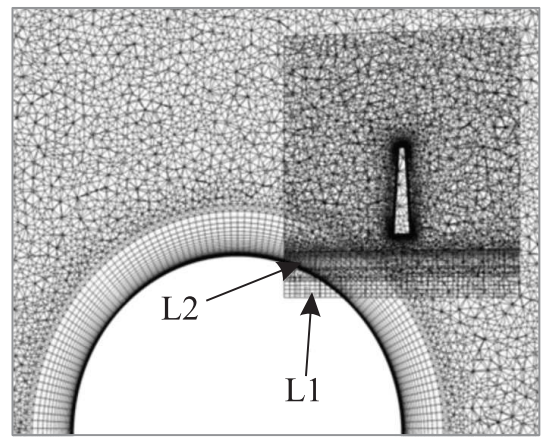

(a)

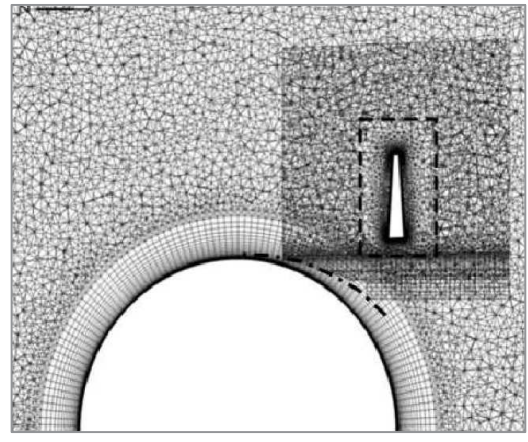

(b)

Figure 5 Hole-cutting chimera technique: (a) without hole-cutting and $(b)$ with hole 
grid points are blanked within the holes. In Fig. $5 b$, the hole for the SB is marked with a dashdotted line and the hole for the $\mathrm{AD}$ by a dashed line.

The hybrid grids were generated with Centaur ${ }^{\mathrm{TM}}$. For all viscous walls, the distribution of $y^{+}$shows values $<1$. In front of the slender body nose, the SB grid has a fine resolution. This is done to resolve the shock, the shear layer, and the flow separation properly. Since the geometry is symmetric with respect to the pitching plane and the oncoming flow has no side slip, only a mesh for a half-model is generated and simulated.

\section{NUMERICAL APPROACH}

The current study has been performed with the DLR TAU-code [28]. TAU is solving the steady and unsteady, three-dimensional (3D), time-accurate RANS equation using a finite-volume formulation on hybrid unstructured grids. Algebraic, one- and two-equation turbulence models, Reynolds stress models (RSM), and explicit algebraic RSM (EARSM) are implemented. To save simulation time in the current study, the one-equation Spalart-Allmaras turbulence model is used as it is implemented in TAU [29]. In preliminary studies at $\mathrm{M}=1.41$, this turbulence model showed good results with respect to a closed separation about the interspace between the disk and the SB nose compared to the experiment of [26]. The same result could be achieved with a Wilcox $\mathrm{k}-\Omega$ turbulence model [30]. Simulations with a shear stress transport (SST) model showed a "retarded" separation [5]. For flux discretization, a second-order AUSMDV upwind scheme is used. The discretization in time is done by an explicit Runge-Kutta scheme. The viscous walls of the disk and the frame of the AD were defined as laminar while the vanes and the SB were defined as turbulent. In the experiment, the disk consists of a porous material to increase the effectiveness of the aerodisk [31]. For simplification, this was neglected in the numerical simulations.

The flow condition of the lowest tested Mach number in [26] was chosen for the numerical investigation: $\mathrm{M}=1.41, \mathrm{Re}=290000$, and $T_{0}=315 \mathrm{~K}$. A smaller Mach number provides a larger physical time step for constant $T_{0}$. A convergence study with respect to time provided a time step of $\Delta t=0.0001 \mathrm{~s}$. In the convergence study, the physics in the separation were also taken into account. The time step must be small enough to ensure that the time interval to overcome the distance between separation and reattachment is discretized several times. This depends on the flow velocity and these, in turn, on the Mach number and $T_{0}=315 \mathrm{~K}$. For all aerodynamic coefficients and the Reynolds numbers, the missile diameter $d=0.036 \mathrm{~m}$ is used as reference length. The pitching motion of the SB was defined with a python script. An interface in TAU makes it possible to define an arbitrary motion of a body via python. The induced flow field due to the motion is considered in the (U)RANS equation of the TAU-solver [32]. As in the experiments, the motion is sinusoidal with a frequency of $f=5 \mathrm{~Hz}$ 


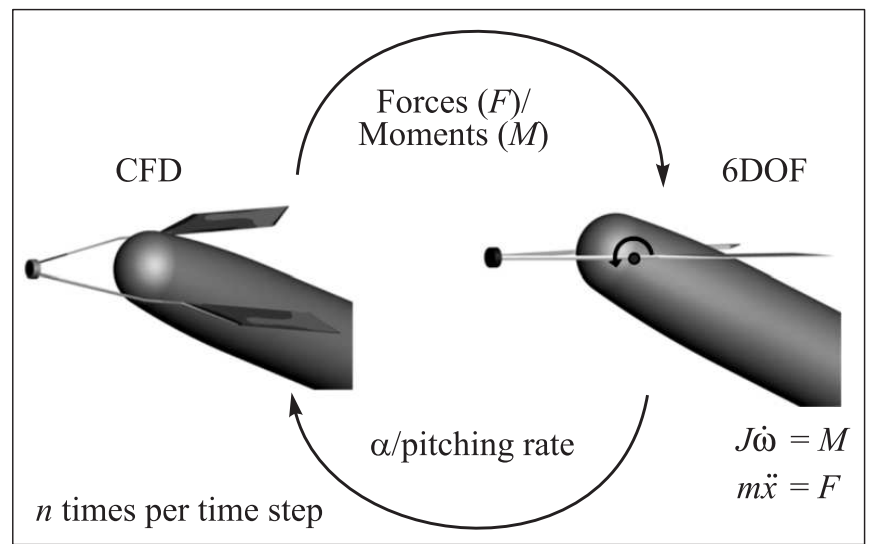

Figure 6 Flow diagram of the coupling between the flow solver and the FM-tool

and an amplitude of $10^{\circ}$ resulting in a range of angle of attack of $0^{\circ}<\alpha<20^{\circ}$. In the wind tunnel experiment [26], the pivot point of the pitching motion was downstream of the missile rear. Even though only the forebody of the wind tunnel model is simulated, the same kinematic relations (distance of the nose to the pivot) as in the experiment are applied.

In contrast to the motion of the $\mathrm{SB}$, the $\mathrm{AD}$ motion is induced by aerodynamic forces. Therefore, a flight mechanic (FM) tool is necessary. This tool is coupled with the flow solver (Fig. 6). Only a pitching of the AD around the rotation axis is possible because of the bearing of the $\mathrm{AD}$ on the experimental model (see Fig. 3). Hence, the other degrees of freedoms are blocked in the FM-tool. The FM-tool takes the aerodynamic forces and the inertia tensor as input values. The FM-tool calculates the pitching rate and a new $\alpha$ of the AD using the equation of motion. The FM-tool and the flow solver are coupled several times per physical time step (see Fig. 6). The exact number of coupling cycles can be defined by the user. Before each interchange of the Input data and the Output data a user specified number of inner iterations is done on both the solver side and the flight mechanic side. A more detailed description of the FM-F-tool for TAU can be found in [32]. In general, it uses the approach of [33].

\section{PRELIMINARY INVESTIGATIONS}

Before the self-aligning-aerodisk concept was numerically investigated on a pitching slender body, the quality of the chimera grid was tested. Therefore, a standard grid without the chimera technique was generated for the same geometry that is assembled with the chimera grid (see Fig. 4c). The flow has been simulated on both grids for the flow condition defined in section 3 except the Mach 


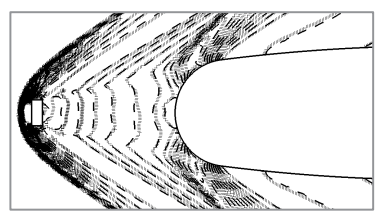

(a)

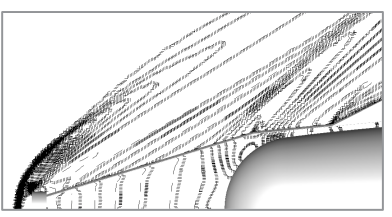

(b)

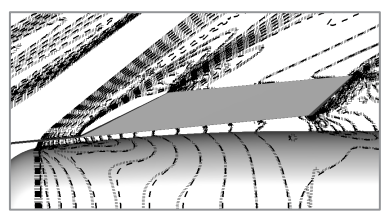

(c)

Figure 7 Comparison of results of simulations with chimera technique and a standard grid (dashed curves): (a) symmetry plane; $(b)$ yawing plane; and $(c)$ yawing plane at vanes

number was 2.2. The $\mathrm{AD}$ was aligned with the $\mathrm{SB}$ for the test case and both aligned with the oncoming flow $\left(\alpha=0^{\circ}\right)$. In Fig. 7, pressure isoclines are shown for both simulations in the symmetry plane and in the plane rectangular to it (yawing plane). The dashed curve corresponds to the simulation on the standard grid. At the gap between the aerodisk and the nose, the interpolation over the shock and within the separation does not lead to large deviations of the isolines. In front of the SB nose, the isolines are more or less coincident again. At the small interspace between the wing and the SB, the agreement of both solutions is even better (Fig. $7 c$ ). The investigation showed a good agreement between the chimera grid and the standard grid. The solution is not changed due to the interpolation even at locations where the physics is quite complicated. No reflection or refraction of the shock at the chimera boundaries is observable.

\section{RESULTS}

The simulation of the pitching slender body with a self-aligning aerodisk was restarted from a solution with an angle of attack of the $\mathrm{SB} \alpha_{\mathrm{SB}}$ and the $\mathrm{AD} \alpha_{\mathrm{AD}}$ of zero. From this condition, the coupling of the flow solver and the FM-tool was started and the pitch-up motion of the SB initiated.

For higher angles of attack, the $\mathrm{AD}$ was not perfectly aligned to the oncoming flow $\left(\alpha_{\mathrm{AD}}=0^{\circ}\right)$ as in the experiment [26] (Fig. 8a). The alignment of the AD is strongly dependent on the reliability of the coupling of the flow solver with the FM-tool. Hence, $\alpha_{\mathrm{AD}}$ was chosen as indicator for the coupling quality. In Fig. $8 a, \alpha_{\mathrm{AD}}$ is plotted against $\alpha_{\mathrm{SB}}$ for two and a half periods. The coupling of the flow solver and the FM-tool gained a reproducible solution after the first period. A variation in time step, number of coupling cycles, and inner iterations for the third period did not change the misalignment.

The total drag coefficient $C_{D}$ is converged between the first and the second periods (Fig. $8 b$ ). It can be concluded that a small variation in $\alpha_{\mathrm{AD}}$ does not result in significant changes in the drag coefficient. The drag coefficient in Fig. $8 b$ shows a trend similar to a hysteresis due to the pitching motion. The pitching motion induces a velocity component $v_{i}$ along the pitching body $\left(v_{i}=\omega r\right)$. 


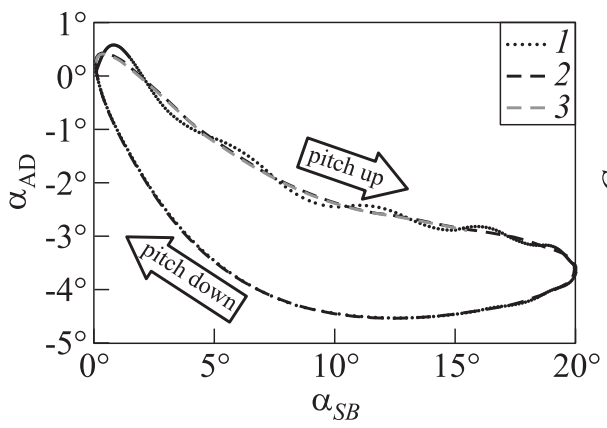

(a)

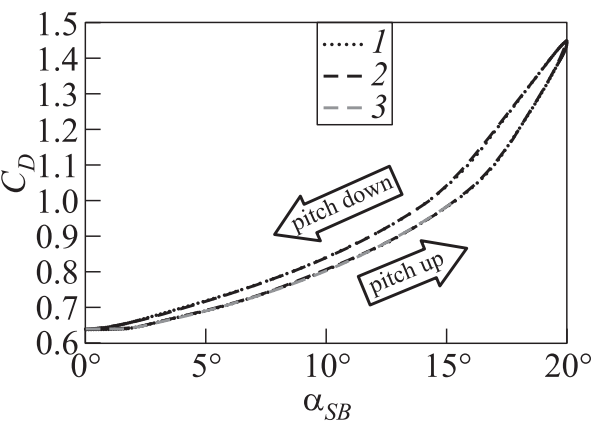

(b)

Figure 8 Time dependency of $\alpha_{\mathrm{AD}}(a)$ and total drag coefficient of the model $C_{D}(b)$ vs. $\alpha_{\mathrm{SB}}: 1-$ period $1 ; 2-$ period 2 ; and $3-$ period 3

Due to the velocity $v_{i}$, the direction of oncoming flow is changed locally. The effective angle of attack $\alpha_{\text {eff }}$ at any position of the roll axis is a superposition of the geometrical angle of attack $\alpha_{\mathrm{SB}}$ and the induced angle of attack $\alpha_{i}$ due to $v_{i}$ (Fig. 9). It means that for a pitch-down maneuver $\alpha_{\text {eff }}$ is larger than $\alpha$ and vice versa for a pitch up maneuver. A larger angle of attack results in a larger $C_{D}$. This explains the trend of $C_{D}$ in Fig. $8 b$.

Figure $8 a$ shows that the self-aligning aerodisk works well for this Mach number and pitching rate. Only a small misalignment of the AD exists which increases with increasing $\alpha_{\mathrm{SB}}$. The experiment [26] observed misalignments of the same order. The misalignment occurs because of the presence of the slender body which deflects the direction of the resulting flow at the vanes. The pressure field near the vanes is similarly influenced by the SB. The AD is "aligned" to this disturbed flow field and not to the oncoming flow.

In Fig. 10, the misalignment is corrected with the induced angle of attack $\alpha_{i}$. The induced angle of attack at the nose tip is used to get the corrected misalignment $\alpha_{\mathrm{AD}, c}\left(\alpha_{\mathrm{AD}, c}=\alpha_{\mathrm{AD}}+\alpha_{i}\right)$. A diminishment of the hysteresis like shape for $\alpha_{\mathrm{AD}, c}$ is observable with increasing $\alpha_{\mathrm{SB}}$. At higher $\alpha_{\mathrm{SB}}$, the vanes of the AD have a larger distance to the SB. An interaction of the vanes and the $\mathrm{AD}$ frame with the boundary layer of the SB is diminished in contrast to smaller $\alpha_{\mathrm{SB}}$. For smaller $\alpha_{\mathrm{SB}}$, this interaction in combination with $v_{i}$ could be responsible for the hysteresis of $\alpha_{\mathrm{AD}, i}$ and the dependence of the misalignment on the pitching direction (upwards/downwards).

In Fig. 11, a schlieren photograph of the experiment is compared with a density gradient plot at the symmetry plane of the simulation for $\mathrm{M}=1.41$ and $\alpha_{\mathrm{SB}}=17.7^{\circ}$ (pitch-up). The agreement of both results is very good. The dominant flow features are captured in the CFD. In addition to the misalignment of $\mathrm{AD}$, the absence of the reattachment of the shear layer on the windward side is in good agreement between experiment and simulation (see Fig. 11). 


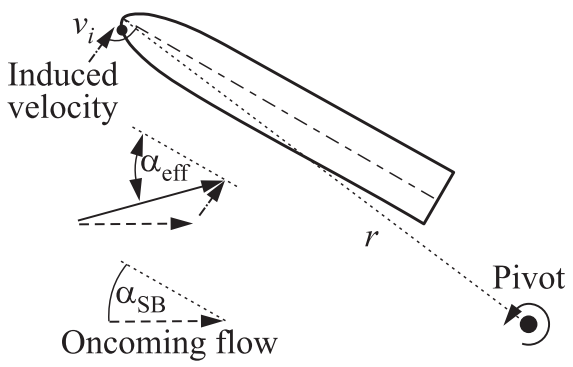

Figure 9 Sketch of the flow on a pitching SB

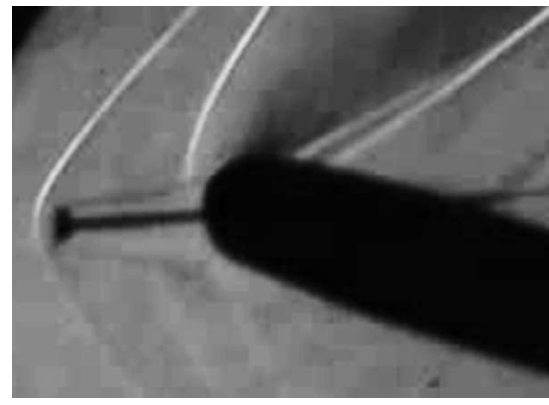

(a)

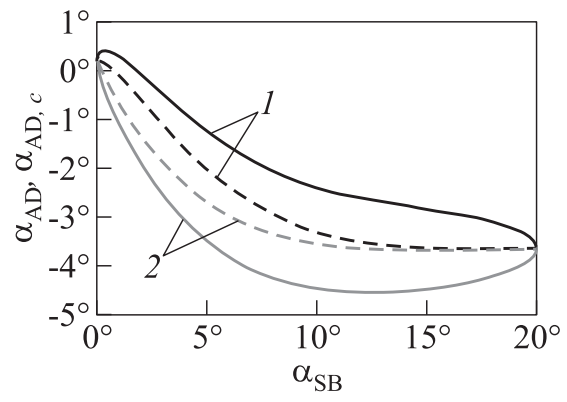

Figure 10 Dependences $\alpha_{\mathrm{AD}}$ vs. $\alpha_{\mathrm{SB}}$ (black curves) and $\alpha_{\mathrm{AD}, c}$ vs. $\alpha_{\mathrm{SB}}$ (grey curves): 1 - pich-up; and 2 - pich-down

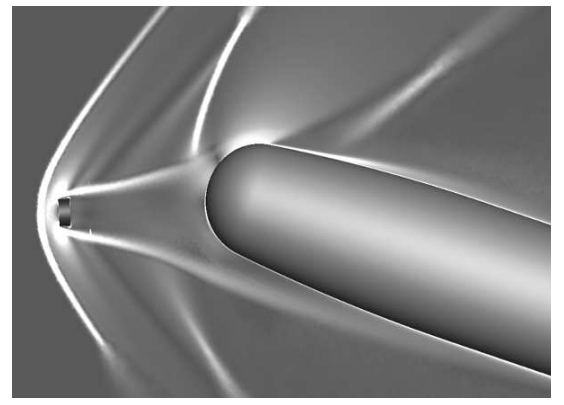

(b)

Figure 11 Comparison of experimental $(a)$ and numerical $(b)$ results. Schlieren photography and density gradients in the symmetry plane (CFD) for $\mathrm{M}=1.41$ and $\alpha=17.7^{\circ}$ (pitch-up)

Additional static simulations at $\alpha_{\mathrm{SB}}=17.7^{\circ}$ have been carried out to analyze the impact of the misalignment and the partial absence of shear layer reattachment. For one simulation, "ideal case," the AD was perfectly aligned $\left(\alpha_{\mathrm{AD}}=0^{\circ}\right)$. For the other simulation, "real case," $\alpha_{\mathrm{AD}}$ was identical with the misalignment of the $\mathrm{AD}$ in Fig. $11 a\left(\alpha_{\mathrm{AD}}=3.06^{\circ}\right)$. Because both are the static simulations, no induced velocity exists. In the ideal case, a circular reattachment line indicating a closed separation can be seen in Fig. 12. The grey field streamlines in Fig. 13 show the presence of a closed separation which is not perfectly rotationally symmetric. For the real case, a complicated 3D flow is indicated by the surface streamline pattern in Fig. 12. On the windward side, the reattachment line vanishes. A pressure increase, seen in the real case due to reattachment, is missing there. Significant higher $c_{p}$ values exist on the upper half of the hemispherical nose (downwind side) at reattachment for the real case. The reason for the 
larger $c_{p}$ is due to a shift of the reattachment line further to the nose tip (see Fig. 12). The angle between the shear layer and the body surface is larger and a larger reattachment angle creates a larger pressure increase at reattachment.

The flow for the real case within the separation is not bounded by a closed separation line and can escape on the windward side. Due to the high pressure at reattachment, the flow is forced to stream towards the symmetry plane and downstream in direction of the arrow in Fig. 13. See also the surface streamlines in Fig. 12. This flow forms a vortex that is responsible for the displacement of the shear layer on the windward side (Fig. 14). The effect of the misalignment is an increase of the total drag at the nose by about $8 \%$ with respect to the ideal case. For the total drag of the SB, an increase of about $4 \%$ occurs with respect to the ideal case. Even considering these problems, a decrease of $C_{D}$ of $\approx 9 \%$ is achieved with respect to the $\mathrm{SB}$ without $\mathrm{AD}$.

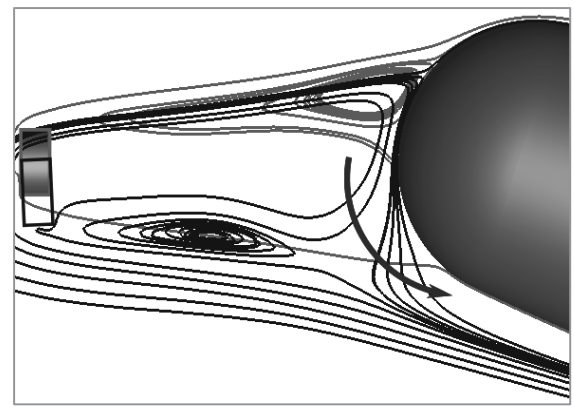

Figure 13 Streamlines in the symmetry plane (grey curves - ideal case and black curves - real case)

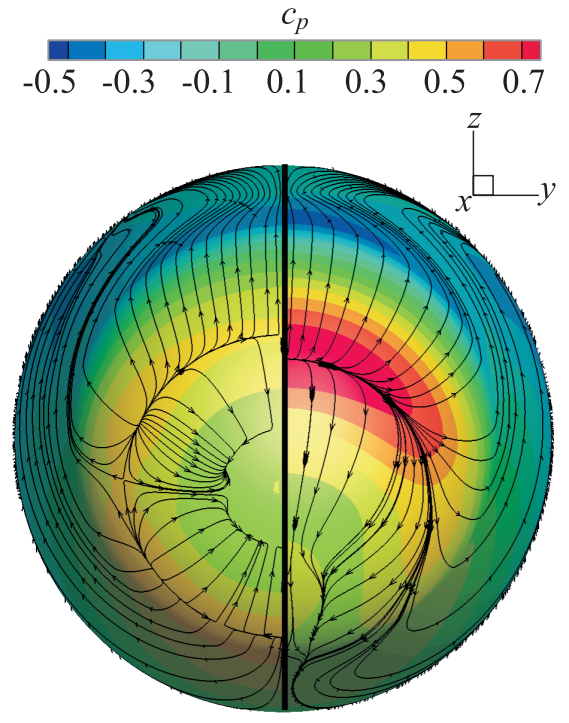

Figure 12 Effect of the misalignment on the pressure distribution at $\mathrm{M}=1.41$ and $\alpha_{\mathrm{SB}}=17.7^{\circ}$ (left side - ideal case and right side - real case)

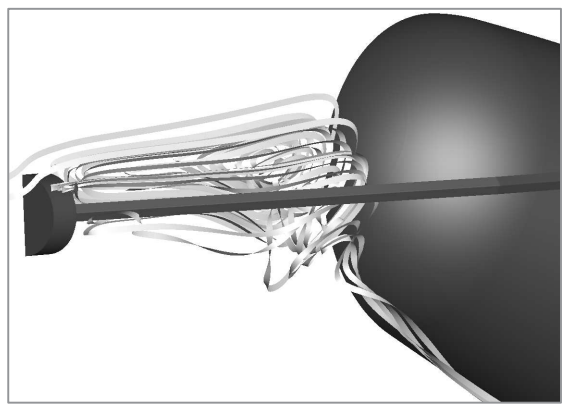

Figure 14 Visualization of the shear layer displacing vortex on windward side of the real case 


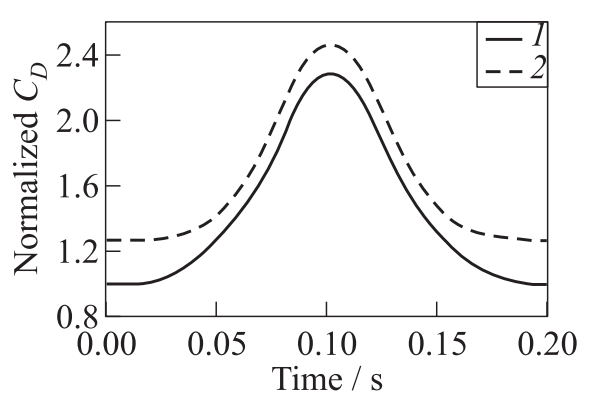

Figure 15 Drag reduction due to the AD (1 - without AD; and 2 - with AD). All drag values are normalized with the drag of the SB with $\mathrm{AD}$ at $\alpha=0^{\circ}$

In Fig. 15, the total drag of a pitching SB with a self-aligning aerodisk is compared with the total drag of an $\mathrm{SB}$ without an aerodisk. The drag is normalized with the drag of the SB with $\mathrm{AD}$ at $\alpha_{\mathrm{SB}}=0^{\circ}$ and $\alpha_{\mathrm{AD}}=0^{\circ}$. The drag can be reduced with the selfaligning aerodisk over the total range of $\alpha$. The beneficial effect of the aerodisk on the drag of an SB is successfully extended to high angles of attack. Although the Mach number is quite small, a decrease in drag of $6.5 \%$ can be achieved at $\alpha_{\mathrm{SB}}=20^{\circ}$. With increasing Mach number, the beneficial effect of a self-aligning aerodisk should be increased even further considering the experiments of $[4,26]$. As expected, the largest decrease in drag is at $\alpha_{\mathrm{SB}}=0^{\circ}$ the lowest at $\alpha_{\mathrm{SB}}=20^{\circ}$. At $\alpha_{\mathrm{SB}}=20^{\circ}$, the decrease in drag is lower because of the misalignment of the $\mathrm{AD}$ and the increasing drag of the cylindrical part with increasing $\alpha$. Even though a misalignment exists, it is small enough to prevent an Edney type IV shock interaction in the total range of $\alpha$.

\section{CONCLUDING REMARKS}

A device to reduce the wave drag of a slender body also at high angle of attack and in maneuvering flight was investigated numerically. An aerodisk was mounted on a frame with vanes and this "self-aligning" aerodisk could pitch about the nose of a slender body. The aerodisk self-aligned to the oncoming flow due to aerodynamic lifting surfaces (vanes). To investigate the self-alignment numerically, the flow solver was coupled with a flight mechanic tool. The device was tested on a slender body pitching sinusoidally with a frequency of $5 \mathrm{~Hz}$ in the range of $0^{\circ} \leq \alpha \leq 20^{\circ}$ at $\mathrm{M}=1.41$. For this test condition, experimental data exist and comparison can be made with the results of the simulations. Additionally, numerical simulations of a slender body without a self-aligning aerodisk have been performed.

The investigation showed that the coupling of the flow solver and the flight mechanic tool gained reproducible and reasonable results. Even in maneuvering flight at high angle of attack, the self-aligning aerodisk followed the oncoming flow. Small misalignments of the aerodisk with respect to the oncoming flow appeared for higher $\alpha$. These misalignments are a result of the disturbance of the flow due to the slender body and the aerodisk itself. The numerical investigation 
showed that even for the Mach number of $\mathrm{M}=1.41$, a decrease in total drag is achievable for the total range of angle of attack. A qualitative comparison of the shock structure and the separation size between the experiment and the numerical investigation showed good agreement. A misalignment of the aerodisk was also observable in the experiment and was of the same order as in the numerical simulation. Based on this work, the complete missile configuration used in [26] will be simulated and quantitatively compared. It will be interesting to see how the AD performs for higher repetition rates and Mach numbers.

\section{REFERENCES}

1. Eggers, A. J., M. Meyer, D. Resnikoff, and H. Dennis. 1956. Bodies of revolution having minimum drag at high supersonic airspeeds. NACA Technical Report TN 1306. 555-566.

2. Takovitskii S. A. 2005. Analytical solution in the problem of constructing axisymmetric noses with minimum wave drag. Fluid Dyn. 41(2):308-312.

3. Allen, H. J., and A. J. Eggers. 1957. A study of the motion and aerodynamic heating of ballistic missiles entering the Earth's atmosphere at high supersonic speeds. NACA Technical Note 4047. Report 1381. 1125-1140.

4. Schülein, E. 2008. Wave drag reduction approach for blunt bodies at high angles of attack: Proof-of-concept experiments. AIAA Paper No. 2008-4000.

5. Chang, P. K. 1970. Separation of flow. New York: Pergamon Press. 777 p.

6. Alexander, S. R. 1947. Results of tests to determine the effect of a conical windshield on the drag of a bluff body at supersonic speeds. NACA RM No. L6K08a.

7. Bogdonoff, S. M., and I. E. Vas. 1959. Preliminary investigations of spiked bodies at hypersonic speeds. J. Aerosp. Sci. 26(2):65-74.

8. Tret'yakov, P. K., A.F. Garanin, G. N. Grachev, V.L. Krainev, A.-G. Ponomarenko, V.N. Tishchenko, and V.I. Yakolec. 1996. Control of supersonic flow around bodies by means of high-power recurrent optical breakdown. Dokl. Phys. 41(11):566-567.

9. Schülein, E., A. A. Zheltovodov, E. A. Pimonov, and M. S. Loginov. 2010. Experimental and numerical modeling of the bow shock interaction with pulse-heated air bubbles. Int. J. Aerospace Innov. 2(3):183-205.

10. Kim, J.-H., A. Matsuda, T. Sakai, and A. Sasoh. 2011. Wave drag reduction with acting spike induced by laser-pulse energy deposition. AIAA J. 49:2076-2078.

11. Schülein, E., and A. Zheltovodov 2011. Effects of steady flow heating by arc discharge upstream of non-slender bodies. Shock Waves 21:383-396.

12. Artem'ev, V. I., V.I. Bergel'son, I. V. Nemchinoy, T. I. Orlova, V. A. Smirnov, and V. M. Khazins. 1989. Change of regime in supersonic flow past an obstacle preceded by a thin channel of reduced density. Fluid Dyn. 24(5):779-784.

13. Meyer, B., H.F. Nelson, and D.W. Riggins. 2001. Hypersonic drag and heattransfer reduction using a forward-facing jet. J. Aircraft 38(4):680-686.

14. Hayashi, K., S. Aso, and Y. Tani. 2005. Numerical study of thermal protection system by opposing jet. 43th AIAA Aerospace Sciences Meeting and Exhibit. 
15. Gordeev, V.P., A. V. Krasil'nikov, V.I. Lagutin, and V. N. Otmennikov. 1996. Experimental study of the possibility of reducing supersonic drag by employing plasma technology. Fluid Dyn. 31(2):313-317.

16. Fomin, V.M., A.A. Maslov, N.D. Malmuth, V.P. Fomichev, A.P. Shashkin, T. A. Korotaeva, A. N. Shiplyuk, and G. A. Pozdnyakov. 2002. Influence of a counterflow plasma jet on supersonic blunt-body pressures. AIAA J. 40(6):1170-1177.

17. Shang, J.S. 2002. Plasma injection for hypersonic blunt-body drag reduction. AIAA J. 40(6):1178-1186.

18. Georgievskii, P. Y., and V. A. Levin. 1988. Supersonic flow past bodies in the presence of external heat release sources. Pisma Zh. Tekh. Fiz. 14(8):684-687.

19. Myrabo, L. N., and Yu.P. Raizer. 1994. Laser-induced air spike for advanced transatmospheric vehicles. 25th AIAA Plasmadynamics and Lasers Conference Proceedings. 13.

20. Guvernyuk, S. V., and A. B. Samoilov. 1997. Control of supersonic flow around bodies my means of a pulsed heat source. Tech. Phys. Lett. 23:333-336.

21. Knight, D., Y.F. Kolesnichenko, V. Brovkin, and D. Khmara. 2008. High speed flow control using microwave energy deposition. 46th AIAA Aerospace Sciences Meeting and Exhibit.

22. Ahmed, M. Y. M., and N. Qin. 2011. Recent advances in the aerothermodynamics of spiked hypersonic vehicles. Prog. Aerosp. Sci. 47(6):425-449.

23. Stalder, J. R., and H.V. Nielsen. 1954. Heat transfer from a hemisphere-cylinder equipped with flow-separation spikes. NACA TN 3287.

24. Edney, B. 1968. Anomalous heat transfer and pressure distribution on blunt bodies at hypersonic speeds in the presence of an impinging shock. Flygtekniska Försöksanstalten FFA. Report 115.

25. Schülein, E. 2010. Flying object for transonic or supersonic flight. Patent U.S. $7,7775,480 \mathrm{~B} 2$.

26. Wysocki, O., E. Schülein, and C. Schnepf. 2014. Experimental study on wave drag reduction at slender bodies by a self-aligning aerospike. New results in numerical and experimental fluid mechanics. Switzerland: Springer International Publishing. 9:583-590.

27. Madrane, A., A. Raichle, and A. Stürmer. 2004. Parallel implementation of a dynamic overset unstructured grid approach. 3rd Conference (International) on Computational Fluid Dynamics (ICCFD3) Proceedings. 733-740.

28. Schwamborn, D., T. Gerhold, and R. Heinrich. 2006. The DLR TAU-Code: Recent application in research and industry. European Conference on Computational Fluid Dynamics (ECCOMAS CFD).

29. DLR. 2012. Technical Documentation of the DLR TAU-Code. Release 2012.1.0.

30. Wilcox, D. C. 1998. Turbulence modeling for CFD. La Canada, CA, USA: DCW Industries. $540 \mathrm{p}$.

31. Schülein, E. 2010. Shock-wave control by permeable wake generators. AIAA Paper No. 2010-4977.

32. Heinrich, R., and A. Michler. 2009. Unsteady simulation of the encounter of a transport aircraft with a generic gust by CFD flight mechanics coupling. CEAS 2009 European Air and Space Conference.

33. Murman, S. M., M. J. Aftosmis, and M. J. Berger. 2003. Simulations of 6-dof motion with a cartesian method. AIAA Paper No. 2003-1246. 